\title{
Photonic Generation of Reconfigurable Orders Ultrawideband Signals by Using Cascaded RSOAs
}

\author{
Hanlin Feng, Shilin Xiao, Lilin Yi, and Weisheng $\mathrm{Hu}$
}

\begin{abstract}
We experimentally demonstrate an ultrawideband (UWB) signals generation scheme with reconfigurable orders using cascaded reflective semiconductor optical amplifiers. Through controlling the gain saturation effect of this cascaded structure, a quasi-high-pass radio frequency filter with tunable cutoff frequency is realized. After passing through this filter, the coded Gaussian like pulse is converted to first-, second-, and third-order UWB signals. All achieved UWB signals accord with the Federal Communications Commission regulations and the third-order UWB signal can eliminate interference with other wireless communications in low frequency range.
\end{abstract}

Index Terms-Microwave filters, semiconductor optical amplifiers (SOAs), ultrawideband (UWB), differentiation.

\section{INTRODUCTION}

$\mathbf{U}$ LTRAWIDEBAND (UWB) signal is considered as one of the most promising scheme for next generation short range wireless communication systems with advantages of high bit rate, low power consumption and high immunity to multipath fading. Federal communications commission (FCC) has defined the spectral density of UWB signal is lower than $-41.3 \mathrm{dBm} / \mathrm{MHz}$ from $3.1 \mathrm{GHz}$ to $10.6 \mathrm{GHz}$. Due to such low power density, the communication range of UWB systems is restricted to several meters. Combing fiber optics with UWB systems can greatly extend the network coverage, so photonic generation UWB signal has become a research focus [1]. UWB signals are categorized into monocycle signal (first order derivative), doublet signal (second order derivative), triplet signal (third order derivative) and other high orders derivative signals. Compared with monocycle and doublet UWB signal, triplet UWB signal has lower power density in low frequency range $(0 \mathrm{GHz}-3 \mathrm{GHz})$ and this may avoid interference with global position system (GPS) band $(0.96 \mathrm{GHz}-1.61 \mathrm{GHz})$ [2]. According to existing research reports, photonic triplet UWB signal generation schemes can be classified into utilizing $\mathrm{N}$ tap radio frequency (RF) filter [3], incoherent summation [4], and integration two complementary doublet UWB pulses [5]. Meanwhile, the cross phase modulation (XPM) effect of

Manuscript received July 1, 2013; revised December 27, 2013; accepted February 28, 2014. Date of publication March 5, 2014; date of current version April 10, 2014. This work was supported in part by the National Nature Science Foundation of China under Grants 61271216, 61090393, and 60972032, in part by the National 973 Project of China under Grants 2010CB328205, 2010CB328204, and 2012CB315602, and in part by the National 863 Hi-Tech Project of China.

The authors are with the State Key Laboratory of Advanced Optical Communication Systems and Networks, Department of Electrical Engineering, Shanghai Jiao Tong University, Shanghai 200240, China (e-mail: fenghanlin@ sjtu.edu.cn; slxiao@sjtu.edu.cn; lilinyi@sjtu.edu.cn; wshu@sjtu.edu.cn).

Color versions of one or more of the figures in this letter are available online at http://ieeexplore.ieee.org.

Digital Object Identifier 10.1109/LPT.2014.2309934

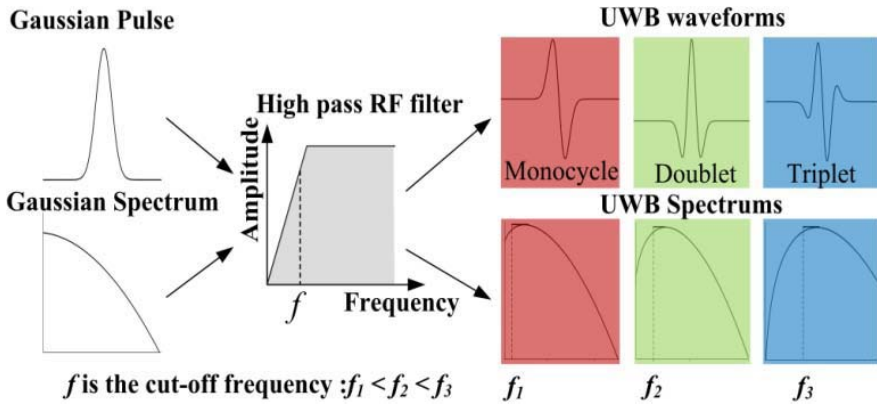

Fig. 1. Principle of first, second and third order UWB signals generation based on high pass RF filter.

integrated semiconductor optical amplifier Mach-Zehnder interferometer structure (SOA-MZI) has been developed to generate triplet UWB signal [6], which is more stable and flexible due to the photonic integration technology.

In this letter, we design and validate a UWB signal generation scheme with reconfigurable differential orders based on cascaded reflective semiconductor optical amplifiers (RSOAs). It is worth noting that gain saturation effect of RSOA has already been used to generate monocycle UWB pulse [7]. In our scheme, gain saturation effect is controlled by adjusting bias currents of two discrete RSOAs. Thus this cascaded RSOAs structure is converted to a quasi-high-pass RF filter with tunable cut-off frequency. After passing through this filter, the low frequency part of Gaussian like pulse sequences is reconfigurable filtered out and different orders UWB signals can be obtained. So this quasi-high-pass RF filter is equivalent to a reconfigurable differentiator with different orders in optical domain. We measure waveforms and electrical spectrums of UWB signals after 5-km SMF transmission. Attributed to single wavelength operation in our proposal, there is few spectrum and waveform distortion observed.

\section{PRinciple And Experimental Setup}

The principle of generation reconfigurable orders UWB signals is shown in Fig. 1. In electrical domain, Gaussian like pulse train is filtered by a high pass RF filter with cut-off frequency $f_{c}$ to suppress low frequency part and pass high frequency part. Through changing the cut-off frequency $f_{c}$ of this filter, various orders Gaussian like pulses are produced. The lowest cut-off frequency $f_{1} \mathrm{GHz}$ corresponds to the first order derivative (monocycle UWB signal). After increasing cut-off frequency to $f_{2} \mathrm{GHz}\left(f_{1}<f_{2}\right)$, doublet UWB signal is obtained by second order differential operation. To further increasing cut-off frequency to $f_{3} \mathrm{GHz}\left(f_{1}<f_{2}<f_{3}\right)$, third order derivative (triplet UWB signal) is generated. 


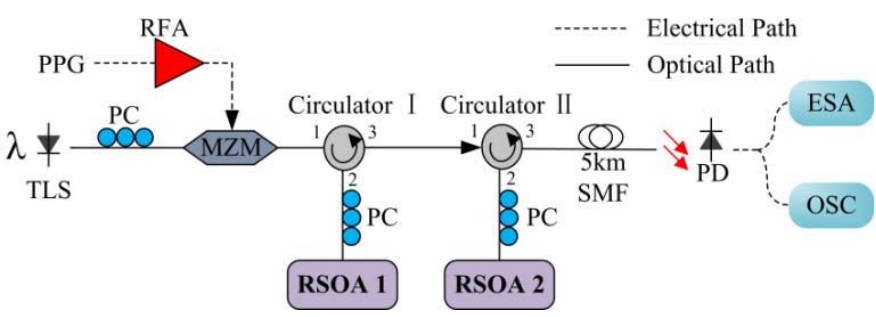

Fig. 2. Experimental setup of reconfigurable orders UWB signals generation scheme.

In reference [8], high-pass RF filter is realized by utilizing gain saturation effect in single semiconductor optical amplifier (SOA). The cut-off frequency $f_{c}$ of high pass RF filter can be roughly estimated as

$$
f_{c} \propto \frac{1}{2 \pi \tau_{\mathrm{c}}}
$$

Note that cut-off frequency $f_{c}$ is inversely proportional to carrier lifetime $\tau_{\mathrm{c}}$. Through increasing input power and bias current, SOA is working in gain saturation region and carrier density in active area is enhanced. However, carrier lifetime decreases with the enhancement of carrier density in active area. This process leads to increasing the cut-off frequency $f_{c}$ of high pass RF filter. After carrier lifetime $\tau_{\mathrm{c}}$ has been determined, the low frequency response of SOA is approximately equal to formula (2).

$$
\mathrm{H}(\mathrm{w})=\mathrm{A} \cdot \mathrm{w}^{2}
$$

A is a constant which related to $\tau_{\mathrm{c}}$. After pass through SOA, ideal Gaussian pulse is converted to equation (3) and the frequency response of second order derivative is generated.

$$
\mathrm{Y}(\mathrm{w})=\mathrm{H}(\mathrm{w}) \cdot \mathrm{X}(\mathrm{w})=\mathrm{A} \cdot \mathrm{w}^{2} \cdot \mathrm{e}^{-\frac{(2 \pi \mathrm{w} \sigma)^{2}}{2}}
$$

The experimental setup is shown in Fig. 2. The lightwave $\lambda$ at $1540 \mathrm{~nm}$ with $6 \mathrm{dBm}$ output power is generated by tunable laser source (TLS). The Gaussian like pulse train is coded by pulse pattern generator (PPG). The output data rate of PPG is $12.5 \mathrm{~Gb} / \mathrm{s}$ with a word length of $2^{31}-1$, by using the regular coded pattern "1000 000000000000 " to represent one and pattern "0000 000000000000 " to represent zero. The repetition rate of generated UWB signal is $781.25 \mathrm{MHz}$. The Mach-Zehnder modulator (MZM) is driven by the coded pulse train amplified by a radio frequency amplifier (RFA). Then by using optical circulator I and optical circulator II, the cascaded RSOAs structure is injection locked by this Gaussian like pulse train. Because RSOA is a polarization sensitive device, the polarization state of injected light is needed to be set by polarization controller (PC) before entering RSOA. The output signals are sent to a photo diode (PD) for photoelectric conversion after 5-km SMF transmission. These receiving signals are analyzed by oscilloscope (OSC) and electrical spectrum analyzer (ESA).

\section{EXPERIMENTAL RESULTS AND DISCUSSION}

We measured the gain saturation and frequency response curve of the cascaded RSOAs structure. The property of single RSOA is used for comparison, as shown in Fig. 3(a) and (b).

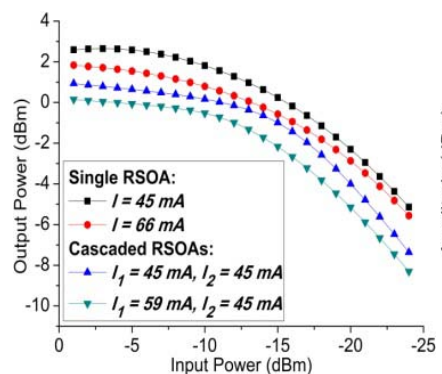

(a)

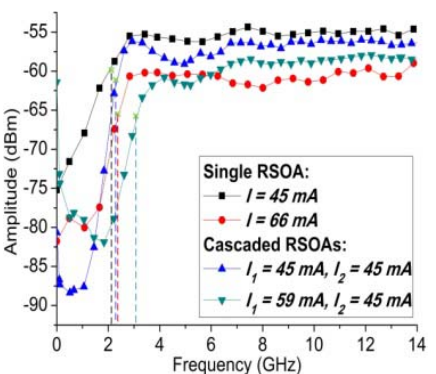

(b)
Fig. 3. Gain saturation and frequency response curves. (a) Gain saturation curve; (b) frequency response curve.

TABLE I

MeAsured CUt-off Frequency and Differential ORDER OF QUASI-HIGH-PASS FILTER

\begin{tabular}{lcl}
\hline Bias Currents & Cut-off Frequency & Order \\
\hline $45 \mathrm{~mA}($ Single RSOA) & $2.25 \mathrm{GHz}$ & First \\
\hline $66 \mathrm{~mA}($ Single RSOA $)$ & $2.5 \mathrm{GHz}$ & Second \\
\hline $45 \mathrm{~mA}\left(I_{1}\right), 45 \mathrm{~mA}\left(I_{2}\right)$ & $2.46 \mathrm{GHz}$ & Second \\
\hline $59 \mathrm{~mA}\left(I_{I}\right), 45 \mathrm{~mA}\left(I_{2}\right)$ & $3.3 \mathrm{GHz}$ & Third \\
\hline
\end{tabular}

Due to the limit of carrier lifetime, gain saturation effect of SOA is only sensitive to certain RF frequencies under $f_{c} \mathrm{GHz}$ [8]. Because of enhanced gain saturation effect, we utilize the cascaded RSOAs structure to realize adjustable high-pass filtering effect. With this enhanced gain saturation effect, the carrier density of cascaded structure is increased which leads to a reduced carrier lifetime. Compared with single RSOA, cascaded structure has a better low frequency filtering effect due to this reduced carrier lifetime. As shown in Fig. 3(a), the cascaded structure has lower output power than single RSOA. By increasing bias current of the first stage, the gain saturation effect can be further enhanced. In Fig. 3(b), the output signal is suppressed by RSOA, when the signal frequency is lower than $f_{c}$. While the signal frequency is higher than $f_{c}$, the output signal is amplified by RSOA.

Through comparison of frequency response curve, the cascaded structure has better filtering effect in low frequency area. In single RSOA configuration, gain saturation effect is limited. When bias current is $45 \mathrm{~mA}$, the first order UWB signal (monocycle) is generated. In Table I, the corresponding cut-off frequency $f_{c}$ is $2.25 \mathrm{GHz}$ and the first order differentiator is realized by this high-pass filter. As bias current changed to $66 \mathrm{~mA}$, the second order UWB signal (doublet) is generated with cut-off frequency of $2.5 \mathrm{GHz}$ and the filter's slope is increased corresponding to the second order differentiator. In the cascaded structure, gain saturation effect is significantly enhanced. The second derivative doublet UWB signal is produced with bias currents of RSOA 1 and RSOA 2 both set to $45 \mathrm{~mA}$, and the corresponding cut-off frequency is $2.46 \mathrm{GHz}$ with better band-stop performance in low frequency area. At this time, this cascaded structure can also be used as a second order differentiator. When bias current of RSOA 1 is increased to $59 \mathrm{~mA}$ and bias current of RSOA 2 remains unchanged, the third order UWB signal (triplet) is obtained. Owing to the increased power of input light, RSOA 2 is working in deep saturation region with 3.3 $\mathrm{GHz}$ cut-off frequency and the third order differentiator 
(a)

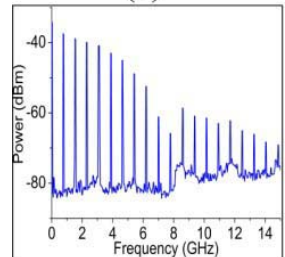

(b)

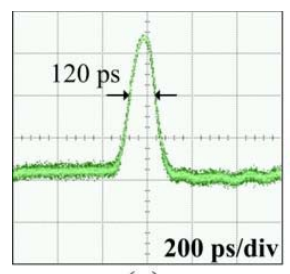

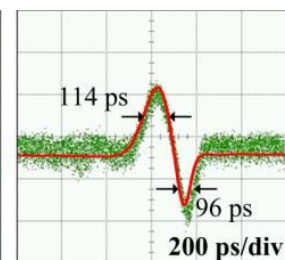

(c)

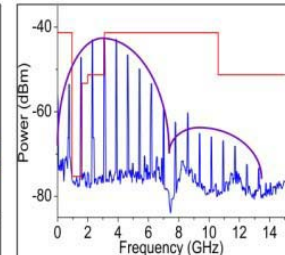

(d)

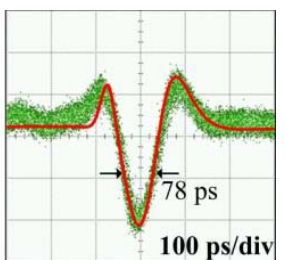

(e)

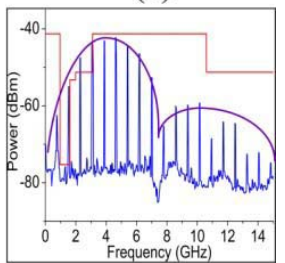

(f)
Fig. 4. Waveforms and electrical spectrums of Gaussian like pulse (a), (b), Monocycle UWB signal (c), (d) and Doublet UWB signal (e), (f) generated by single RSOA.

is achieved. On the contrary, if we decrease the bias current of RSOA 1, the amplification factor of the first stage will reduce. So, with a lower injection power from the former stage, the gain saturation effect of RSOA 2 will decreased. The corresponding cut-off frequency of this cascaded structure is also reduced and the generated UWB signal will be degraded to previous derivative orders. However, if we further increase the bias current of RSOA 2, the gain saturation and cut-off frequency of this cascaded structure will not be improved significantly. It's mainly due to the limited carrier density of RSOA 2. In this case, the generated UWB waveform is decided by the input power from the first stage.

The waveforms and spectrums of Gaussian like pulse and generated UWB signals are shown in Figs. 4 and 5 with theoretical waveforms (red line) and spectrums (violet line). Fig. 4(c) and (d) depict the waveform and spectrum of monocycle UWB signal which generated by the quasi-highpass filter with $2.25 \mathrm{GHz}$ cut-off frequency $(I=45 \mathrm{~mA}$, single RSOA). This monocycle UWB signal has full width at half maxium (FWHM) about 114 ps with central frequency of $3.1 \mathrm{GHz}$ and $10 \mathrm{~dB}$ bandwidth of $5.2 \mathrm{GHz}$ which corresponding to a fraction bandwidth of $168 \%$. Fig. 4(e) and (f) demonstrate the waveform and spectrum of doublet UWB signal which generated by a quasi-high-pass filter with $2.5 \mathrm{GHz}$ cutoff frequency ( $I=66 \mathrm{~mA}$, single RSOA). This doublet UWB signal has FWHM about 78 ps with $3.8 \mathrm{GHz}$ central frequency and $10 \mathrm{~dB}$ bandwidth of $5.3 \mathrm{GHz}$ which corresponding to a fraction bandwidth of $139 \%$.

Fig. 5(a) and (b) show the waveform and spectrum of reversed doublet signal generated by cascaded RSOAs, when bias currents $I_{1}$ and $I_{2}$ are both set to $45 \mathrm{~mA}$. Though the cutoff frequency $(2.46 \mathrm{GHz})$ is very close to that of single RSOA with $66 \mathrm{~mA}$ bias current, the cascaded structure has steeper slope which equivalent to a second order differentiator. So a polarity reversed doublet UWB signal is produced. This doublet UWB signal has FWHM about 76 ps with $4.6 \mathrm{GHz}$ central frequency and $10 \mathrm{~dB}$ bandwidth of $5.1 \mathrm{GHz}$, corresponding to fraction bandwidth of $111 \%$. Therefore, as cut-off frequencies are basically the same, polarity reversed signals are produced by high-pass filter with steeper slope. However, for achieving

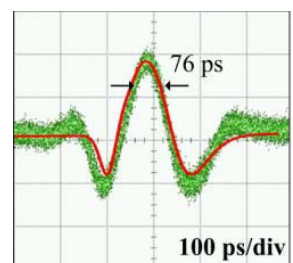

(a)

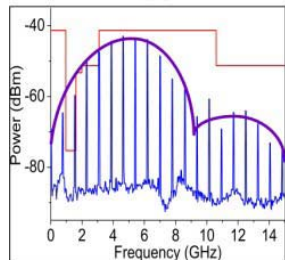

(b) (c)

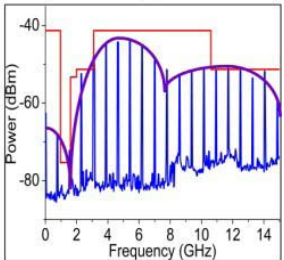

(d)

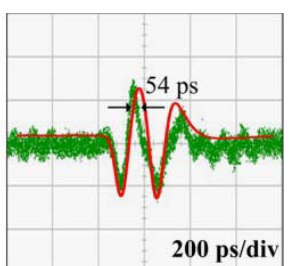

Fig. 5. Waveforms and electrical spectrums of Doublet UWB signal (a), (b) and Triplet UWB signal (c), (d) generated by cascaded RSOAs.

higher order UWB signal, cut-off frequency has to be enlarged. Fig. 5(c) and (d) manifest waveform and spectrum of triplet UWB signal which generated by a quasi-high-pass filter with $3.3 \mathrm{GHz}$ cut-off frequency $\left(I_{1}=59 \mathrm{~mA}, I_{2}=45 \mathrm{~mA}\right)$. This triplet UWB signal has FWHM about 54 ps with central frequency of $4.5 \mathrm{GHz}$ and $10 \mathrm{~dB}$ bandwidth of $11 \mathrm{GHz}$ which corresponding to a fraction bandwidth of $245 \%$. The third order triplet UWB has $-72 \mathrm{XdBm}$ power dip from $1 \mathrm{GHz}$ to $1.6 \mathrm{GHz}$ which can effectively avoid interference with other wireless communications, especially the GPS service in $1.57542 \mathrm{GHz}$.

\section{CONCLuSion}

In conclusion, a novel reconfigurable orders UWB signals generation scheme based on cascaded RSOAs is proposed. The generated first, second and third order UWB signals have very good performance. The proposed scheme is simple, colorless and single wavelength operation, which may become a bridge between fixed and wireless access networks.

\section{REFERENCES}

[1] J. Yao, "Photonics for ultrawideband ommunications," IEEE Microw. Mag, vol. 10, no. 4, pp. 82-95, Jun. 2009.

[2] X. Fu, Y. Dai, and C. Shu, "Reconfigurable photonic ultrawideband pulse generation from an optically injected semiconductor laser," Opt. Lett., vol. 38, no. 6, pp. 968-970, 2013.

[3] M. Bolea, J. Mora, B. Ortega, and J. Capmany, "Optical UWB pulse generator using an $\mathrm{N}$ tap microwave photonic filter and phase inversion adaptable to different pulse modulation formats," Opt. Express, vol. 17, no. 7, pp. 5023-5032, 2009.

[4] Z. Enbo, X. Xing, L. King-Shan, and K. K.-Y. Wong, "A powerefficient ultra-wideband pulse generator based on multiple PMIM conversions," IEEE Photon. Technol. Lett., vol. 22, no. 14, pp. 1063-1065, Jul. 15, 2010.

[5] L. Pengxiao, C. Hongwei, W. Xu, Y. Hongchen, C. Minghua, and X. Shizhong, "Photonic generation and transmission of 2-Gbit/s powerefficient IR-UWB signals employing an electro-optic phase modulator," IEEE Photon. Technol. Lett., vol. 25, no. 2, pp. 144-146, Jan. 15, 2013.

[6] V. Moreno, M. Rius, J. Mora, M. A. Muriel, and J. Capmany, "Integrable high order UWB pulse photonic generator based on cross phase modulation in a SOA-MZI," Opt. Express, vol. 21, no. 19, pp. 22911-22917, 2013.

[7] G. Chen and S. Pan, "Photonic generation of ultrawideband signals based on frequency-dependent gain saturation in a reflective semiconductor optical amplifier," Opt. Lett., vol. 37, no. 20, pp. 4251-4253, 2012.

[8] K. Sato and H. Toba, "Reduction of mode partition noise by using semiconductor optical amplifiers," IEEE J. Sel. Topics Quantum Electron., vol. 7, no. 2, pp. 328-333, Mar./Apr. 2001. 